\title{
Autologous Hamstring Tendon Graft Augmentation in Acute Midsubstance Patellar Tendon Rupture: Case Report and Review of Literature
}

\section{Shafiq Hackla*, DS Solanki, IPS Oberoi and Satvir Singh}

Department of Orthopaedics, Artemis Hospital, Gurgaon

*Corresponding Author: Shafiq Hackla, Department of Orthopaedics, Artemis Hospital, Gurgaon.
Received: March 04, 2020

Published: March 12, 2020

(c) All rights are reserved by Shafiq Hackla., et al.

\section{Abstract}

Patellar tendon rupture is common cause of extensor mechanism disruption in young patients. It usually occurs in partially flexed knee with foot planted. Patellar tendon rupture occurs most commonly at inferior pole of patella or avulsion from tibial tuberosity. Patellar tendon mid substance rupture is a very rare injury. We found only two case reports of mid substance rupture in literature. We reported a case of mid substance patellar tendon rupture in a 48 year old male.

Keywords: Midsubstance Rupture; Hamstring Graft; Patellar Tendon

\section{Case Report}

A 48 years old male presented with history of pain in front of knee while dancing and falling backward. There was history of popping sound and inability to walk after the injury.

On examination of knee, there was swelling of knee and palpable defect in the inferior pole of patella (Figure 1). The patella was superiorly migrated and extensors lag was present. Radiograph showed superiorly migrated patella (patella Alta). MRI of knee reveal mid substance rupture of patellar tendon and collection of hematoma in front of knee joint (Figure 2). The patient was immobilised in knee immobilizer and prepared for surgery after anesthetic check up.

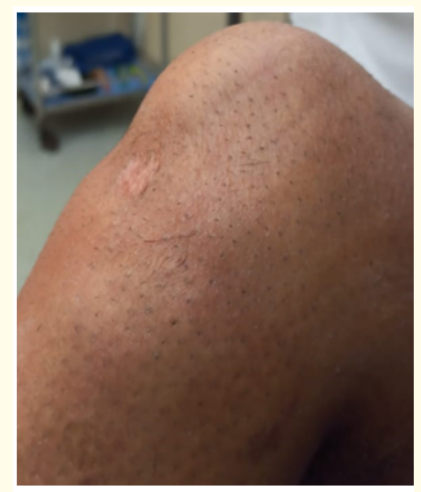

Figure 1: Shows the depression below the patella indicating patellar tendon discontinuity.

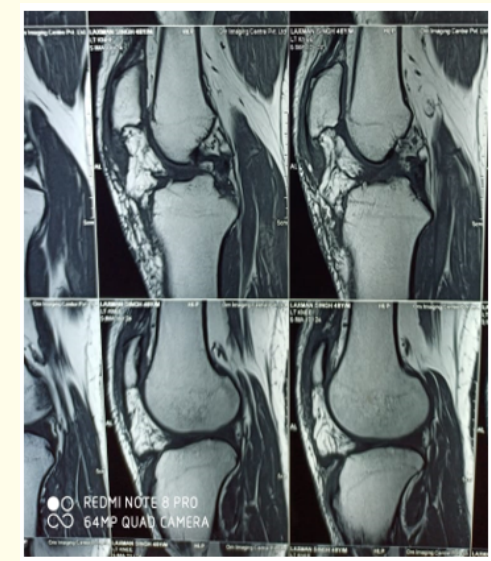

Figure 2: Shows Saggital MRI revealing superiorly migrated patella with patellar mid substance tendon rupture and collection of hematoma behind the tendon and in front of knee joint

The patient was operated in spinal anesthesia with Supine position and tourniquet applied. Midline incision starting from superior pole of patella to tibial tuberosity with distal curving of incision to medial side of tuberosity. Mid substance rupture of patellar tendon identified (Figure 3). Margins of tendon debribed and sutured with locking krackow sutures in 60 degree knee flexion (Figure 4). 


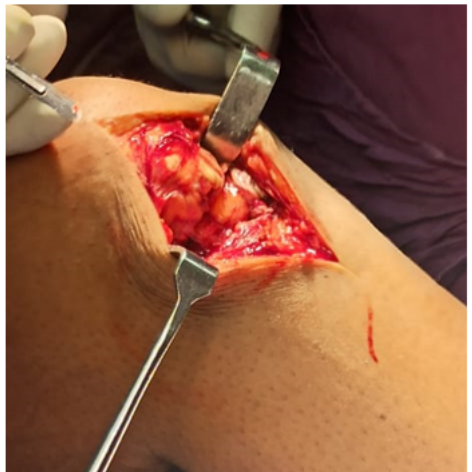

Figure 3: Showing incision from superior pole of patella to tibial tuberosity. There is tendon discontinuity in the middle of patella revealing mid substance rupture.

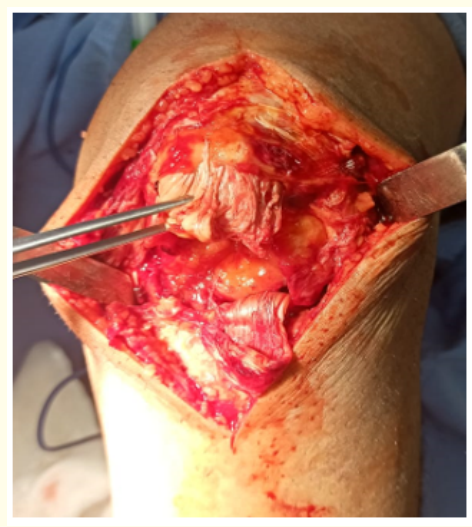

Figure 4: Shows the two ends of patellar tendon with fraying and degenerative changes in two ends of tendon.

Hamstring tendons harvested from the ipsilateral leg. Two tunnels made, one in middle of patella transversely and other $2 \mathrm{~cm}$ below and behind the tuberosity (Figure 5). Harvested tendon passed from medial to lateral from tibial tuberosity, and lateral to medial in patella (Figure 6). The tendon is then sutured back to itself on medial side. The reinforced tendon is then augmented with suture tapes (Figure 7). The strength of repair was checked intraoperatively while flexing and extending the knee. The wound was closed in layers and above knee slab applied with knee extension.

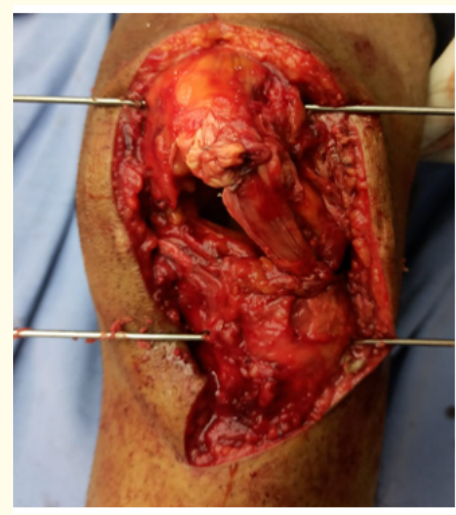

Figure 5: Shows the debridement of edges of tendon and repair with krackow stitches. Tibial and patellar tunnels in the bone are made and prepared for augmentation with the autograft.

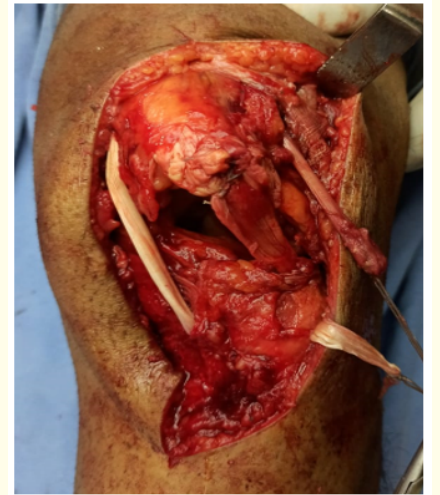

Figure 6: Shows the passing of hamstring autograft from lateral to medial in the patella and medial to lateral in tibial tuberosity.

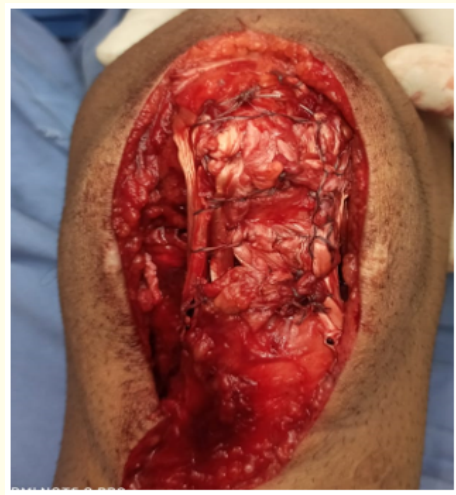

Figure 7: Shows the two ends of graft are sutured upon itself and reinforcement of repair with suture tapes.

\section{Discussion}

Rajani., et al. reported case of bilateral patellar mid substance rupture in case of total knee arthroplasty. The patient had taken multiple steroid injections prior to knee replacement. They attributed it to steroid injection for mid substance rupture [11]. Yousef., et al. found one case mid substance patellar tendon in a pediatric patient [5]. So far only two cases of mid substance patellar tendon rupture found in literature and no case of primary rupture in middle age patients.

Rupture of patellar tendon involves a complete tear of the tendon anywhere from its origin on patella inferior pole to its insertion on tibial tuberosity. Tendon ruptures usually occur in middle age male patients. High tensile forces coupled with weak tendon predisposes to ruptures. The patellar tendon is responsible for extending the knee and helps in walking [1]. The deficiency in extensor mechanism limits patient ambulation [2]. The extensor mechanism disruption mostly occurs due to patella fracture. Tendon ruptures are less common but the location and type of tendon rupture has an important bearing on the management of these ruptures by surgical intervention [3].

Patellar tendon rupture occurs in patients with long-standing chronic tendon degeneration [4]. The mechanism of rupture is contraction of quadriceps muscle in flexed knee. Running on stairs, fall from height, suddenly changing direction are some of mechanism 
for the injury. The most common site for patellar tendon rupture is inferior pole of patella. Tibial tuberosity avulsion and mid substance ruptures can also occur in tendon [5]. The strain is high at inferior pole of patella that is why most ruptures occurs at inferior pole.

The treatment of rupture include conservative treatment with immobilisation of knee in full extension and exercise program. Operative treatment includes primary repair or reconstruction of the tendon [6]. The indication for tendon repair is complete rupture in which tendon ends can be approximated without tension [7]. The type of repair depends upon location of the tear. The mid substance tear are repaired by suturing the ends of tendon with each other. Bone tunnels are made when it involves either the the inferior pole or tibial tuberosity. Sutures anchors also have been used in repairing the tendon ruptures. Repair of the tendon has also been reinforced with auto graft or allograft when the tendon isnot healthy and there are adhesions in tendon or tissue is friable. The fear of degeneration and loss of excursion in tendon make it more important to treat tendon repair with a sense of urgency. There are various techniques described for patellar tendon reconstruction $[9,10]$. We performed primary repair of tendon along with augmentation by autologous hamstring tendon graft and suture anchor [11]. First, the patellar tendon is repaired with an end-to-end technique and the semitendinous tendon is harvested. Two bone tunnels, one at the tibial tubercle and another tunnel in middle of patella made. The hamstring graft passed through the tunnels and sutured upon itself. The graft is sutured along the length of the patellar tendon on both margins in tension at $60^{\circ}$ of knee flexion. Intra-operative imaging was performed to assess the patella height. This technique provides a significant augmentation of patellar tendon [12].

\section{Conclusion}

Patellar mid substance rupture has not reported much in the literature. Unusual injury mechanism and tendon degeneration in a middle age person predisposes to this kind of rupture. These patients can be managed with primary repair and reconstruction with semitendinosus auto graft. Good functional outcome can be expected in these patients.

\section{Bibliography}

1. Redler A., et al. "Rupture of the Patellar Tendon After Platelet-Rich Plasma Treatment: A Case Report". Clinical Journal of Sport Medicine 30.1 (2020): e20-e22.

2. Alqasim E., et al. "Total patellectomy with extensor mechanism reconstruction following pathological fracture due to patellar Ewing's sarcoma". BMJ Case Reports (2018).

3. Behery OA., et al. "Combined Tibial Tubercle Fracture and Patellar Tendon Avulsion: Surgical Technique and Case Report". Journal of Orthopaedic Case Reports 8.3 (2018): 18-22.
4. Morton S., et al. "Patellar Tendinopathy and Potential Risk Factors: An International Database of Cases and Controls". Clinical Journal of Sport Medicine 27.5 (2017): 468-474.

5. Yousef MAA. "Combined avulsion fracture of the tibial tubercle and patellar tendon rupture in pediatric population: case series and review of literature". European Journal of Orthopaedic Surgery and Traumatology 28.2 (2018): 317-323.

6. Ali Yousef MA and Rosenfeld S. "Acute traumatic rupture of the patellar tendon in pediatric population: Case series and review of the literature". Injury 48.11 (2017): 2515-2521.

7. Camarda L., et al. "Bilateral ruptures of the extensor mechanism of the knee: A systematic review". Journal of Orthopaedics 14.4 (2017): 445-453.

8. Courtney PM., et al. "Is There Any Role for Direct Repair of Extensor Mechanism Disruption Following Total Knee Arthroplasty?" The Journal of Arthroplasty 33.7 (2018): S244-S248.

9. Woodmass JM., et al. "Patellar Tendon Repair With Ipsilateral Semitendinosus Autograft Augmentation". Arthroscopy Techniques 6.6 (2017): e2177-e2181.

10. Harato K., et al. "Surgical Technique to Bring Down the Patellar Height and to Reconstruct the Tendon for Chronic Patellar Tendon Rupture". Arthroscopy Techniques 6.5 (2017): e1897e1901.

11. A Rajani., et al. "Bilateral Spontaneous Midsubstance Patellar Tendon Rupture after Bilateral Total Knee Arthroplasty". Journal of Orthopaedic Case Reports 6.2 (2016): 75-77.

12. H Van der Bracht., et al. "Augmentation of a patellar tendon repair with an autologous semitendinosus graft". Acta Orthopaedica Belgica 75 (2009): 417-419.

13. J Espregueira-Mendes., et al. "Augmentation of Patellar Tendon Repair with Autologous Semitendinosus GraftdPorto Technique". Arthroscopy Techniques 6.6 (2017): e2271-e2276.

\section{Assets from publication with us}

- Prompt Acknowledgement after receiving the article

- Thorough Double blinded peer review

- Rapid Publication

- Issue of Publication Certificate

- High visibility of your Published work

Website: https://www.actascientific.com/

Submit Article: https://www.actascientific.com/submission.php Email us: editor@actascientific.com Contact us: +919182824667 